\title{
QUANTUM-CONFINED STARK EFFECT AND RECOMBINATION DYNAMICS OF SPATIALLY INDIRECT EXCITONS IN MBE-GROWN GaN-AIGaN QUANTUM WELLS
}

\author{
Pierre Lefebvre*, Bernard Gil*, Jacques Allègre*, Henry Mathieu*, Nicolas Grandjean**, \\ Mathieu Leroux**, Jean Massies**, Pierre Bigenwald *** \\ * Groupe d'Etude des Semiconducteurs - CNRS - Université Montpellier II. \\ Case Courrier 074. 34095 Montpellier Cedex 5, France. \\ **Centre de Recherche sur l' Hétéro-Epitaxie et ses Applications - CNRS \\ Rue B. Grégory. F-06560 Valbonne, France. \\ ***LPM - Université d'Avignon - 33, rue Pasteur. 84000 Avignon, France.
}

Cite this article as: MRS Internet J. Nitride Semicond. Res. 4S1, G3.69 (1999)

\begin{abstract}
We analyze the low-temperature photoluminescence decay times, for a series of MBE-grown samples embedding GaN-AlGaN quantum wells. We investigate a variety of configurations in terms of well widths, barrier widths and overall strain states. We find that not only the wells but also the barriers are submitted to large built-in electric fields. In the case of narrow barriers $(5 \mathrm{~nm})$, these fields favor the nonradiative escape of carriers from narrow wells into wider wells. When all wells have the same width, the field in such narrow barriers allow us to observe the recombination of long-lived "inter-well" excitons at energies close to those of the short-lived "intra-well" excitons. Our results also prove that the energies and the dynamics of excitonic recombinations depend on the parameters of the heterostructures in a complicated way, due to the interplay of piezoelectric and spontaneous polarizations.
\end{abstract}

\section{INTRODUCTION}

Nitride semiconductor quantum wells (QWs) are emerging as promising systems for the realization of blue-UV light emitters and detectors [1,2]. Their importance is such that it is crucial to obtain a better comprehension of mechanisms that rule optical processes in these heterostructures, especially from the dynamical viewpoint. In particular, it has been recently shown [3-8] that some original behaviors are due to the presence, along the growth axis of the QWs, of built-in electric fields of several hundred $\mathrm{kV} / \mathrm{cm}$. Considering the large lattice-mismatch between the constituents, these fields have been assigned [5-8] to the piezoelectric effect, due to very large piezoelectric coefficients. In fact, theoretical investigations $[9,10]$ have established that two mechanisms, essentially similar in nature, should be considered to explain the large electric fields reported thus far. According to these studies, the values of the fields in the wells and in the barriers depend on the difference of piezoelectric polarizations between the two materials and on the difference of their so-called spontaneous (or equilibrium) polarizations.

The latter polarizations are essentially provoked by the nonideal c/a ratio of lattice parameters in the hexagonal elementary cells. The magnitudes of these spontaneous polarizations have been found to be particularly large in group-III nitride semiconductors; in fact, they are the largest of all III-V compounds [9], with values comparable to those for $\mathrm{ZnO}$ or $\mathrm{BeO}$. However, Bernardini and Fiorentini have also calculated that the spontaneous polarizations of $\mathrm{GaN}$ and $\mathrm{InN}$ are not 
very different. Thus, for ideal (disorder-free) InGaN-GaN QWs, the main effect which induces built-in electric fields is the difference of piezoelectric polarizations. On the other hand, this is not the case for the GaN-AlGaN pair, because the spontaneous polarizations of the two compounds are really different. Both spontaneous and piezoelectric contributions have to be considered for GaN-AlGaN QW systems. For ideal infinite GaN/GaAlN superlattices, with well and barrier widths $L_{W}$ and $L_{B}$, the electric fields in the wells and in the barriers are given respectively by $[10,11]$ :

$$
\begin{aligned}
& E_{W}=L_{B} \cdot\left(\left|P^{s p}{ }_{W}\right|-\left|P^{s p}{ }_{B}\right|-\left|P^{p z}{ }_{W}\right|-\left|P_{B}^{p z}\right|\right) /\left(\varepsilon_{B} L_{W}+\varepsilon_{W} L_{B}\right) \\
& E_{B}=L_{W} \cdot\left(\left|P^{s p}{ }_{B}\right|+\left|P^{p z}{ }_{W}\right|+\left|P_{B}^{p z}\right|-\left|P^{s p}{ }_{W}\right|\right) /\left(\varepsilon_{B} L_{W}+\varepsilon_{W} L_{B}\right)
\end{aligned}
$$

where $\varepsilon_{\mathrm{W}}$ and $\varepsilon_{\mathrm{B}}$ are the dielectric constants for the GaN wells (W) and the AlGaN barriers (B). $P^{s p}{ }_{W}$ and $P^{s p}{ }_{B}$ are the spontaneous polarizations which have the same sign in both materials. On the other hand, $P^{p z}{ }_{W}$ and $P^{p z}{ }_{B}$ (the piezoelectric polarizations) have opposite signs, at least for QW systems which are pseudomorphically grown (i.e. lattice-matched) on GaN buffer layers, themselves grown on sapphire substrates (this is the case of our samples). Indeed, GaN layers undergo a small biaxial compression, while $\mathrm{AlGaN}$ layers are under biaxial tension. A first interesting result expressed by these equations is that the field inside the GaN QWs is not directly given only by the piezoelectric effect in the GaN layer. The second interesting result is that this field inside the QWs is a growing function of the barrier width.

In this paper, we present time-resolved photoluminescence (TRPL) experiments which support the above theoretical predictions, in particular in terms of the role played by the barriers in GaN - AlGaN QW systems.

\section{EXPERIMENTS}

The samples were grown by molecular beam epitaxy on sapphire substrates, followed by a $2 \mu \mathrm{m}$ thick GaN buffer layer, grown at $800^{\circ} \mathrm{C}$. Next, GaN/GaAlN multiple- or single- QWs have been deposited, all samples being terminated by $30 \mathrm{~nm}$ thick GaAlN layers, with the same Al composition as the barriers. These barriers have thicknesses of $5 \mathrm{~nm}$ for the two multiple QWs presented and of $30 \mathrm{~nm}$ for the single QW. All samples have been grown in the same setup, under identical growth conditions. Thus, there is no reason to expect important differences between them, in terms of bulk or interface nonradiative processes, for instance.

Sample 1 is a single $\mathrm{GaN} / \mathrm{Ga}_{0.91} \mathrm{Al}_{0.09} \mathrm{~N}$ QW with nominal thickness of $17 \mathrm{MLs}(1 \mathrm{ML}=$ $2.59 \AA$ ). Sample 2 is made of ten $\mathrm{GaN} / \mathrm{Ga}_{0.89} \mathrm{Al}_{0.11} \mathrm{~N}$ QWs having all the same nominal thickness of 6 MLs. Sample 3 contains four $\mathrm{GaN} / \mathrm{Ga}_{0.89} \mathrm{Al}_{0.11} \mathrm{~N}$ QWs with respective thicknesses: 3, 7, 11 and 15 MLs, nominally, from the surface towards the GaN buffer layer.

Detailed results of continuous-wave spectroscopy, at $\mathrm{T}=2 \mathrm{~K}$, are available elsewhere [12-14]. In summary, Sample 1 shows a single PL peak at $3389.3 \mathrm{meV}$, well below the excitonic gap of $\mathrm{GaN}$, due to a strong electric field. The latter induces a very small oscillator strength for the fundamental transition, which explains that no reflectivity feature corresponds to the PL line. Sample 2 exhibits a single intense PL line at $3583.2 \mathrm{meV}$, Stokes-shifted by $\sim 30 \mathrm{meV}$ from the corresponding strong reflectivity structure. The PL lines of the 15, 11, 7 and 3 ML-wide QWs in Sample 1 peak respectively at 3462.0, 3513.3, 3569.0 and $3671.9 \mathrm{meV}$. All PL lines are Stokesshifted from their reflectance structure by $\sim 40-50 \mathrm{meV}$. This is assigned [15] to the localization of excitons on areas where the QWs exceed their nominal thicknesses by 2 MLs. Thus, we have considered such "enlarged" well thicknesses in our envelope function calculations of transition energies. These calculations include excitonic effects and the material parameters have been 
taken from Refs. [16-18]. The magnitude of the electric field in the QWs is a fitting parameter: a very good agreement is found between calculated and measured energies provided that we include fields of $600 \mathrm{kV} / \mathrm{cm}$ for Sample 1 and of $450 \mathrm{kV} / \mathrm{cm}$ for Samples 2 and 3 [13].

Such a difference of internal fields does not result from different strain states. This is proved by the energies of the $\mathrm{A}, \mathrm{B}$ and $\mathrm{C}$ excitons in the $\mathrm{GaN}$ buffers, measured by reflectivity. It is well-known that these energies have a direct correlation [16] with the amount of residual inplane compressive strain in GaN layers grown on sapphire. These energies are respectively, of 3480.0, 3486.5 and 3503.0 meV, for Sample 1, 3478.8, 3484.7 and 3502.0 meV, for Sample 2, and $3482.2,3488.8$ and $3506.4 \mathrm{meV}$, for Sample 3.

We see that the strain in $\mathrm{GaN}$ layers is almost the same for all samples: it is slightly smaller for Sample 2 than for Sample 1, and slightly smaller for Sample 1 than for Sample 3. From these results and assuming that $\mathrm{AlGaN}$ layers are lattice-matched onto the $\mathrm{GaN}$ buffers, we find that the differences of piezoelectric polarizations of wells and barriers are almost identical for the three samples. This is a first evidence that the magnitudes of internal fields are not a simple function of the compressive strain in the GaN layers. Instead, the larger electric field in Sample 1 can be explained by the thicker barriers, according to Eq. (1). Consistently, the "opposite" field in the barriers should be 4-5 times weaker for Sample 1 than for the other samples [see Eq. (2)].

For TRPL experiments, the excitation is provided by laser pulses with $\lambda=270 \mathrm{~nm}$ and with typical temporal width of $2 \mathrm{ps}$, at a repetition frequency of $82 \mathrm{MHz}$. A helium closed-cycle cryostat is used for cooling the samples down to a minimum of $8 \mathrm{~K}$. The PL is analyzed through a spectrometer and detected by a streak camera. The overall time-resolution of the setup is $\sim 5$ ps. Figure 1(a) shows the temporal evolution of the PL spectrum of Sample 1. We find that the decay is multi-exponential in all regions of this spectrum. We get a satisfactory fitting only when including at least three exponential decays. We also notice a red-shift of the line of $\sim 6 \mathrm{meV}$ for increasing times, which suggests some band filling effects and the partial screening of the internal field by photocarriers, at short times.
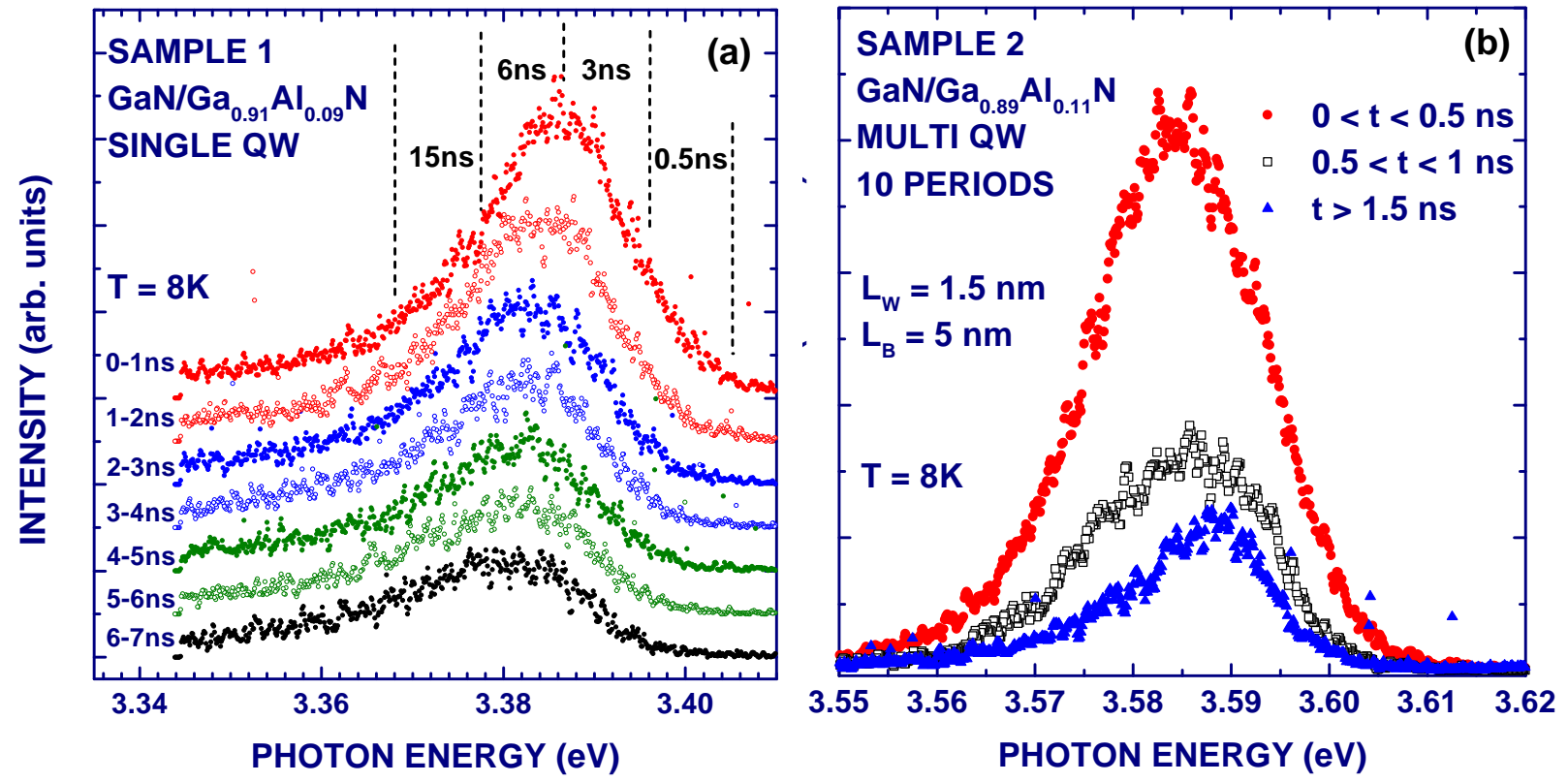

Figure 1: Evolution with time of the PL of Samples 1 (a) and 2 (b), shown by the spectra recorded on various time intervals. Indicative decay times are shown for various spectral ranges.

The overall decay time is of more than $10 \mathrm{ns,} \mathrm{especially} \mathrm{in} \mathrm{the} \mathrm{low-energy} \mathrm{range,} \mathrm{where} \mathrm{it}$ reaches the upper limits of our time detection. Such decay times are fairly comparable with the estimations of $\sim 35 \mathrm{~ns}$ for the radiative lifetime of such localized excitons, that we made via variational envelope-function calculations of the excitonic oscillator strength $[19,20]$, including a 
field of $600 \mathrm{kV} / \mathrm{cm}$. On the other hand, the PL decay times, displayed in Fig. 2, for the QWs of Sample 3 are much shorter than the radiative lifetimes, estimated for a field of $450 \mathrm{kV} / \mathrm{cm}$.

These radiative lifetimes are again of several nanoseconds, and increase almost exponentially with the well width. The experimental decay times rather show a linear increase and are of the order of several tens of picoseconds. Moreover, we have measured here clear mono-exponential decays. We interpret these decays as arising from the competition between radiative recombinations and the nonradiative transfer of carriers across the barriers, from each well into its wider neighbor and, eventually, into the GaN buffer. This interpretation is supported by the linear increase of the times for reaching maximum PL intensity, when going from the narrowest QW (arbitrarily assigned to zero-delay) to the widest QW (see open circles in Fig. 2). Indeed, the latter result strongly suggests a kind of recycling of the carriers that are lost by a well, which cross the barrier and feed the neighboring wider well. This kind of transfer is made possible by the quantum coupling of the different QWs across the thin $(5 \mathrm{~nm})$ barriers. Each electron subband corresponds to a wave function delocalized over the entire multi-QW system. An electron in an excited state, which is mainly localized in a given well, can either recombine radiatively with a hole within this well, or thermalize, by phonon emission, towards a lowerlying state of the entire structure, which happens to be mainly localized on another well. The efficiency of this process is a growing function of the probability of leakage of the envelope function in the adjacent well, which is small in the present case (for the electron state centered on the $3 \mathrm{ML} \mathrm{QW}$, the probability in this well is $\sim 180$ times larger than in the $7 \mathrm{ML} \mathrm{QW})$. If the QWs were strongly coupled, the carriers would thermalize even more efficiently towards their ground state: the recombinations from narrow wells would have even shorter lifetimes. By enhancing the leakage probablility, the strong built-in electric fields in the barriers slightly favor such carrier transfers.

We can only propose a qualitative picture of the electric fields in all layers, because many material parameters are still poorly known. Theoretical calculations indicate that the general band profiles in our samples should resemble those sketched in Fig. 3, but these are only valid for ideal insulators, i.e. band curvatures are likely to occur due to free carriers, in real samples.

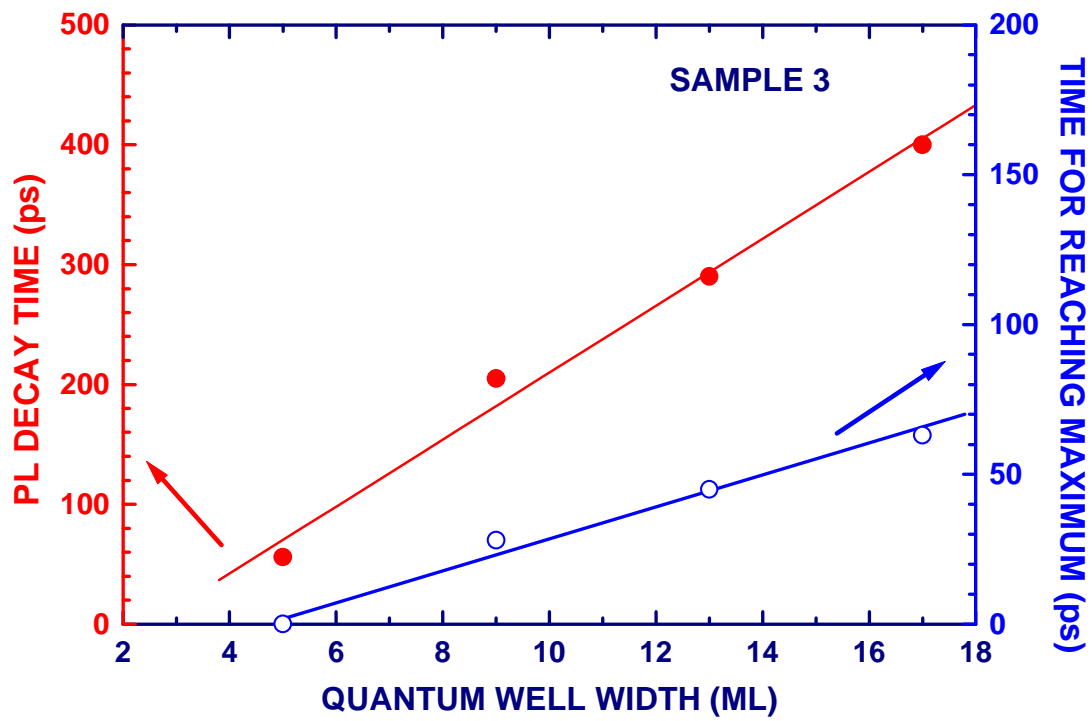

Figure 2: PL decay times (solid circles) versus QW width (nominal widths increased by two units have been used). Open circles show the change of the time for reaching maximum PL intensity. 


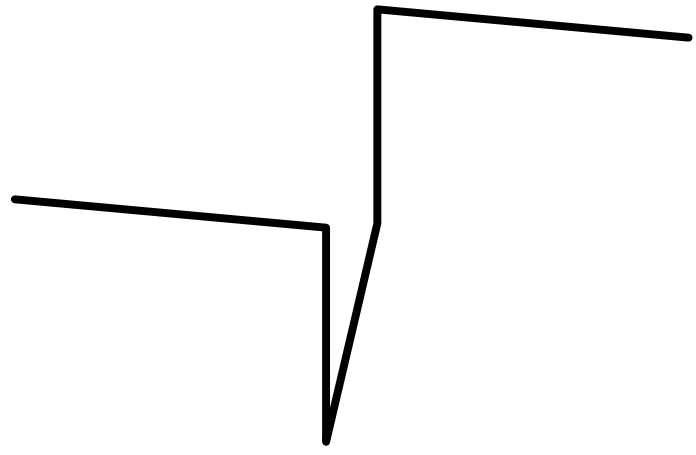

SAMPLE 1

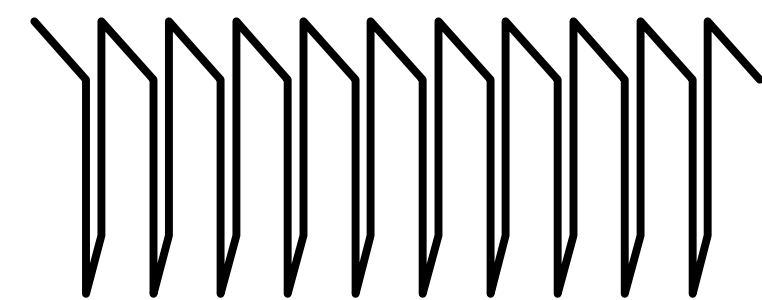

SAMPLE 2

Figure 3: Sketch of conduction band minima in Samples 1 and 2 as deduced from Eqs (1) and (2), in the case of GaN/GaAlN QWs without any free carriers. Reasonable values of fields, widths, and potential discontinuities have been used, and both figures have the same scale.

The case of Sample 3, not shown, should be like the case of Sample 2, except for the varying well widths. We remark that we have assumed, for multi-QWs, an overall "flat" band profile. We have an indication of this lineup by analyzing TRPL results obtained on Sample 2 (see below).

Figure 1(b) shows the change of the PL spectrum of Sample 2 versus time. Contrary to the case of the single QW, we observe a clear blue-shift of $5 \mathrm{meV}$ of the PL maximum between small and large delays. This totally unusual behavior, and the somewhat irregular shape of the spectrum, suggest that this PL line is made of several contributions, due to a variety of configurations in terms of local well and barrier widths. This is confirmed by the multiexponential decay in all regions of the spectrum. Dominant fast components (typical decay times of $200 \mathrm{ps}$ ) are present for the entire line, whereas much slower decays (typically $2.5 \mathrm{ns)}$ are rather present in the high-energy part of the line. We interpret these slow decays as arising from spatially indirect, inter-well exciton recombinations, whereas fast decays rather correspond to excitons recombining within a given well.

Inter-well transitions are favored, here, by the electric fields which push electrons towards the left-hand side of the QW (see Fig. 3), while holes in its left-hand next neighbor are pushed to the right. Nervertheless, intra-well and inter-well recombinations cannot be observed simultaneously without an essential mechanism, namely the strong in-plane localization of electrons and holes [12-15,20,21], with trapping energies of several tens of meV, and localization radii smaller than the Bohr radius [21]. Then, there are statistically some occurences for which the in-plane distance of the localization sites of the electron and the hole is large (compared e.g. to the barrier width). In such cases, an inter-well recombination is more probable than its intra-well counterpart.

Variational calculations of transition energies and oscillator strengths allow us to get a satisfactory description of experimental results: we have performed a series of calculations assuming variations and permutations of well and barrier widths near their nominal values (e.g. between 6 and 9 MLs, for QWs), for a model-system made of three QWs. We find a "band" of short-lived "intra-well" excitons covering the range between 3585 and $3625 \mathrm{meV}$ and two "bands" of inter-well excitons at 3595-3605 meV and 3615-3620 meV. Inter-well excitons lie at larger energies than intra-well excitons mainly because of a smaller binding energy. But it is crucial to note that the energies of the two types of transitions would be completely different if the general band profile was not "globally flat", as shown in Fig. 3. 


\section{CONCLUSION}

We have shown the key role played by the complex interplay of piezoelectric and spontaneous polarizations in GaN-GaAlN QWs, by analyzing time-resolved PL experiments on such samples. This interplay induces a complex dependence of built-in electric fields on layer thicknesses and biaxial strains. Consequently, each sample should be considered individually (a single QW cannot be regarded as a multiple QW) and original behaviors appear, in terms of excitonic energies and lifetimes and of carrier transfer mechanisms. These particularities pave the way for versatile applications of such QWs, which are undoubtedly promised to a bright future.

\section{REFERENCES}

[1] S. Nakamura and G. Fasol, "The Blue Laser Diode" - Springer Verlag Berlin, 1997.

[2] See, for instance "Group III Nitride Semiconductor Compounds", Edited by B. Gil, The Clarendon Press -1998, ISBN 0-19-850159-5.

[3] J. S. Im, J. Off, A. Sohmer, F. Scholtz and A. Hangleiter, Materials Science Forum, 264-268, 1299 (1998).

[4] N. Grandjean and J. Massies, Appl. Phys. Lett. 73, 1260 (1998).

[5] J. S. Im, H. Kollmer, J. Off, A. Sohmer, F. Scholz and A. Hangleiter, Mat. Res. Soc. Symp. Proc. 482, 513 (1998).

[6] H. Amano, T. Takeushi, S. Sota, H. Sakai and I. Akasaki, Mat. Res. Soc. Symp. Proc. 449, 1143 (1997).

[7] K. P. O’Donnell, T. Breitkopf, H. Kalt, W. Van der Stricht, I. Moerman, P. Demeester and P. G. Middleton, Appl. Phys. Lett. 70, 1843 (1997).

[8] J. S. Im, H. Kollmer, J. Off, A. Sohmer, F. Scholz, and A. Hangleiter, Phys. Rev. B 57, R9435 (1998).

[9] F. Bernardini, V. Fiorentini and D. Vanderbilt, Phys. Rev. B 56, R10024 (1997).

[10] F. Bernardini and V. Fiorentini, Phys. Rev. B 57, R9427 (1998).

[11] F. Bernardini and V. Fiorentini, to be published.

[12] P. Lefebvre, B. Gil, J. Allègre H. Mathieu, N. Grandjean, M. Leroux, J. Massies, P. Bigenwald and P. Christol, 24th International Conference on the Physics of Semiconductors, Jerusalem (july 1998). To be published.

[13] M. Leroux, N. Grandjean, M. Laügt, J. Massies, B. Gil, P. Lefebvre and P. Bigenwald, Phys. Rev. B 58, 13371R (1998).

[14] P. Lefebvre, J. Allègre, B. Gil, H. Mathieu, P. Bigenwald, N. Grandjean, M. Leroux and J. Massies, Phys. Rev. B, to be published.

[15] G. Bastard, C. Delalande, M. H. Meynadier, P. M. Frijlink and M. Voos, Phys. Rev. B. 29, 7042 (1984); C. Delalande, M. H. Meynadier and M. Voos, Phys. Rev. B 31, 2497 (1985).

[16] M. Tchounkeu, O. Briot, B. Gil, J. P. Alexis, and R.-L. Aulombard, J. Appl. Phys. 80, 5352 (1996).

[17] M. Suzuki, T. Uenoyama and A Yanase, Phys. Rev. B 52, 8132 (1995).

[18] S.L. Chuang and C.S. Chang, Phys. Rev. B 54, 2491 (1996)

[19] B. Gil and P. Bigenwald, Solid Stat. Commun. 94, 883,( 1995), P. Bigenwald and B. Gil, Phys. Rev. B 51, 9780, (1995).

[20] A. V. Kavokin, Phys. Rev. B 50, 8000 (1994); E. L. Ivchenko, A. V. Kavokin, Sov. Phys. Semicond. 25, 1070 (1991).

[21] P. Lefebvre, J. Allègre, B. Gil, A. Kavokin, H. Mathieu, W. Kim, A.Salvador, H. Morkoç and A. Botchkarev, Phys. Rev. B 57, 9447R (1998). 